Christine Develotte

ENS Lyon, ICAR

cdevelotte@gmail.com

Marie-Anne Paveau

Université Paris 13, Pléiade

ma.paveau@orange.fr

\title{
Pratiques discursives et interactionnelles en contexte numérique. Questionnements linguistiques
}

\begin{abstract}
Résumé
Cet article propose une mise au point sur les travaux menés sur les discours numériques natifs essentiellement en France depuis les années 1990, dans les disciplines liées à la revue Langage et société. On propose d'abord une vue d'ensemble des rapports entre sciences du langage et communication électronique et numérique, dans leurs dimensions discursives, interactionnelles et sociales. On insiste ensuite sur les apports notables des sciences de l'information et de la communication aux traitements linguistiques des données numériques. On formule ensuite des questions que les productions électroniques/numériques posent aux sciences du langage dans leurs dimensions sociolangagières. On décrit enfin, à partir du traitement de quelques exemples, des propositions élaborées actuellement pour rendre compte des spécificités des discours numériques natifs sous l'angle discursif et interactionnel.
\end{abstract}

Mots-clés: analyse du discours numérique, communication électronique, discours numérique natif, interactions médiées par écran, interactions multimodales, corpus numériques.

\section{Discursive and interactive practices in a digital context. Linguistic issues}

Abstract : This article suggests to focus on the studies that had been carried on webnative discourse mainly in France, since the 1990s, in the fields related to the Langage et société journal. We first propose an overview of the established links between Language Sciences and digital electronic communication taking into account their discursive, interactional and social dimensions. We will then emphasize the significant contributions of the Communication Sciences to the linguistic processing assigned to digital data. Afterwards, we express questions that electronic / digital productions raise to Language Sciences, throughout their sociolinguistic dimensions. Based on given examples, we will finally describe current elaborate proposals to account for webnative discourse specificities in terms of discursive and interactional dimensions.

Key words: digital discourse analysis, electronic communication, webnative discourse, screen mediated interactions, multimodal interactions, digital corpora.

\section{Introduction}

La plupart des communications et relations humaines s'élaborent désormais, à des degrés divers selon les contextes géographiques, économiques, politiques et culturels, dans les dispositifs numériques, entendus comme des écosystèmes communicationnels en ligne, à 
partir d'appareils connectés. Cet article voudrait montrer comment certaines des disciplines concernées par la revue Langage et société, en particulier celles qui ont affaire au texte, au discours et à l'interaction (désormais TDI), prennent en compte les pratiques numériques contemporaines qui s'élaborent dans les environnements numériques.

Nous entendons ici numérique au sens large: sont appelés numériques des productions langagières issues d'un appareil informatique en contexte connecté, dans la mesure où elles produisent une relation dans un écosystème technologique. Le terme électronique, que nous emploierons aussi, et qui a été pionnier dans les premières approches linguistiques, est conservé par certains chercheurs pour insister plutôt sur la dimension des appareils et des pratiques (Liénard, Zlitni, 2015) ou désigner des productions hors connexion (certains téléphones, les ordinateurs).

Dans un premier temps, nous brossons à grands traits l'histoire des rapports entre sciences du langage (désormais SDL) et communication électronique et numérique, en insistant sur les dimensions discursives, interactionnelles et sociales, dans les champs anglophones et francophones, étroitement liés sur cette question. Nous montrons ensuite que les sciences de l'information et de la communication (désormais SIC) offrent aux SDL de riches apports pour l'analyse des productions langagières et discursives natives d'internet. Nous évoquons ensuite une série de questions que les productions électroniques/numériques nous semblent poser à la linguistique dans ses dimensions sociolangagières. Nous décrivons enfin, à partir du traitement de deux exemples, les propositions faites actuellement pour rendre compte des spécificités des discours numériques natifs sous l'angle discursif et interactionnel.

\section{Brève histoire des rapports entre les sciences du langage et les productions électroniques/numériques}

Les SDL se sont penchées, dès les années 1990, sur les aspects formels de l'écriture électronique au sein de la Computer Mediated Communication anglophone (CMC) et de la Communication médiée/médiatisée par ordinateur francophone (CMO), puis sur certains aspects discursifs et conversationnels propres au web $1.0^{1}$.

\subsection{CMC et CMO dans le champ linguistique}

Dans un premier temps, les travaux qui se sont inscrits dans une perspective linguistique, tels en France ceux d'Anis (1998) et de Panckhurst (1998) et, aux États-Unis, de Baron (2000) et Crystal (2001), se sont surtout intéressés aux variations « technolinguistiques ${ }^{2}$ de ce que Crystal a appelé " the Netspeak». Aux États-Unis, Susan Herring $(1996,2004)$ a, quant à elle, commencé dès le début des années 1990 à travailler sur des questions discursives (liées à la problématique du genre ou à la politesse par exemple) dans les communications médiées par ordinateur. Elle a été suivie sur ce terrain par des chercheurs français (notamment Fraenkel, Marcoccia dirs 2003, Pierozak 2003).

Ces recherches, centrées sur les marques langagières nouvelles des productions sur les appareils (ordinateurs, téléphones), n'avaient pas pour objet de rendre compte de la façon dont les productions verbales connectées s'articulent à des formes de sociabilité reconfigurées voire inventées en ligne, particulièrement à l'ère du web conversationnel (années 2000). En effet, elles sont souvent restées internalistes, se concentrant sur les formes de langue et de discours, ou proposant des comparaisons entre formes en ligne et formes hors ligne.

Depuis la fin des années 2000, la CMC francophone connaît un renouvellement: sur l'impulsion de Fabien Liénard et Sami Zlitni, est organisé tous les deux ans depuis 2010 un colloque international consacré à la communication électronique. Aux corpus d'origine

\footnotetext{
${ }^{1}$ Rappelons que le web est un service d'internet, au même titre que la messagerie électronique par exemple. Parler des discours d'internet et de ceux du web ne revient donc pas au même.

2 Les linguistes qui se sont montré les plus enclins à étudier ces nouveaux corpus avaient aussi souvent un intérêt marqué pour l'informatique, comme François Mangenot, Rachel Panckurst ou Jean Véronis.
} 
constitués à partir de SMS, de chats, de courriels ou de forums de discussion du web 1.0, se sont ajoutés les terrains du web social et conversationnel : les approches ont donc évolué, faisant se rencontrer les approches internalistes et des approches plus ouvertes sur les contextes sociaux, culturels mais également géographiques (Liénard, Zlitni 2015 dirs). Le téléphone s'étant transformé en appareil multifonction (ordinateur, assistant personnel, appareil photo, borne wifi), Fabien Liénard propose actuellement de parler de CMT, « communication médiée par téléphone ».

\subsection{Approches discursives}

Au milieu des années 2000, "Les discours de l'Internet: nouveaux corpus, nouveaux modèles ? ", numéro des Carnets du Cediscor coordonné par Florence Mourlhon-Dallies, Florimond Rakotonoelina et Sandrine Reboul-Touré, s'appuie sur des corpus textuels. On y trouve aussi bien des études sur le forum de discussion (Michel Marcoccia, Christelle Celik et François Mangenot), la liste de diffusion (Jacques Anis) que sur le chat multilingue (Patrick Chardenet). À ce moment-là de l'histoire de la communication en ligne, le grand public n'avait que peu accès à d'autres types de communication que ceux liés à l'écriture de type asynchrone (forum, méls) ou synchrone (chat). La méthodologie choisie par les coordonnateurs du numéro visait à "faire travailler " sur des corpus électroniques un certain nombre d'"entrées en linguistique de discours qui ont déjà fait leurs preuves sur des supports plus traditionnels" (Mourlhon-Dallies et al. dir. 2004, p. 9). Se trouvent ainsi réunies dans ce même ouvrage, comme cadres d'appui à l'analyse des «discours de l'internet », la complexification du cadre participatif de Goffman (Michel Marcoccia), l'organisation en séquences conversationnelles au regard de l'écoulement temporel (Jacques Anis), la décomposition de l'objet de discours (Patrick Chardenet) en encore le genre de discours (Jean-Yves Colin et Florence Mourlhon-Dallies).

À partir de la fin des années 2000, après l'arrivée du web social, des blogs et des réseaux sociaux numériques (désormais RSN), les analystes du discours entament des recherches sur les productions en ligne, considérées à la fois comme des "corpus sauvages » (Calabrese 2011 dir.) et un " eldorado » (Pierozak 2010). II s'agit de travaux ponctuels, considérant internet et tout particulièrement le web 2.0 comme de nouveaux terrains pour décrire des objets ou des catégories préexistants, à l'aide d'outils théoriques et méthodologiques prénumériques, c'est-à-dire élaborés préalablement sur des corpus hors ligne. Cela explique que les approches soient souvent centrées sur le genre, l'énonciation ou l'argumentation. On citera par exemple Amossy 2011, examinant les formes du consensus dans les commentaires en ligne, ou Maingueneau 2013, exploitant la notion d'hypergenre à partir de ses travaux précédents sur les genres de discours.

Bien qu'envisageant le niveau du discours, et impliquant donc en principe les contextes sociaux, ces travaux peinent cependant à envisager l'ensemble environnemental constitué par les écosystèmes numériques, nécessitant selon nous une approche externaliste : les discours natifs de l'internet, et du web en particulier, présentent des traits proprement technolangagiers et technodiscursifs, coproduits par les scripteurs usagers et les affordances du système. Une analyse du discours numérique fondée sur une approche linguistique écologique, c'est-à-dire intégrant les dimensions technologiques des univers numériques, peut selon nous rendre compte de la complexité des énoncés natifs en ligne dans leurs contextes sociotechniques (Develotte et al. 2011 dirs, Paveau 2015 dir.).

\subsection{Ouvertures anthropologiques et interactionnistes}

En février 2004, le colloque « La communication électronique: Approches linguistiques et anthropologiques " organisé à la Maison des Sciences de l'Homme de Paris, constitue un repère dans l'histoire naissante du domaine. L'ouverture scientifique s'inscrit dans le titre, l'anthropologie étant convoquée pour venir enrichir les approches linguistiques. Différents domaines des SDL sont convoqués: l'anthropologie linguistique, la sémiolinguistique, la pragmatique, la sociolinguistique et l'analyse conversationnelle viennent éclairer des objets 
en cours d'élaboration scientifique comme le montre par exemple, l'émergence de désignations telles que conversation électronique. Signalons également l'approche issue de l'analyse conversationnelle d'inspiration ethnométhodologique de Lorenza Mondada (1999) qui s'est penchée sur l'étude de l'interaction sur Internet en se centrant sur les différentes formes de séquentialité identifiables dans les courriels et les forums de discussion.

Par ailleurs les premiers travaux de Christine Develotte et Thierry Lancien (2000), effectués sur des corpus d'articles d'encyclopédies sur CD-Rom, ont cherché à développer une " analyse des discours multimédias" organisée autour de différents ordres sémiolinguistiques susceptibles de rendre compte de l'enchâssement des trois niveaux que sont « la mise en écran » (modifiable, avec des éléments superposables, déroulables, etc.), " la mise en média » (texte, vidéo, image, audio) et "la mise en discours " (l'énonciation spécifique liée aux caractéristiques de l'espace de communication). En 2011, Christine Develotte, Richard Kern et Marie-Noëlle Lamy publient un collectif destiné à montrer que la conversation en «face à face distanciel » (par MSN) vient revisiter les principes mis au jour pour la conversation en face à face, Décrire la conversation en ligne (Develotte, Kern, Lamy 2011 dir.). La synchronie interactionnelle, par exemple, est indissociable de la qualité du flux numérique, de la distorsion du signal audio ou vidéo et demande un ajustement de la part des interactants. Parmi les spécificités repérées dans les conversations en ligne, on retiendra la coexistence d'espaces différents (Marcoccia 2011), les chevauchements de parole plus nombreux qu'en présentiel (Traverso 2011), l'ordinateur comme acteur social dans l'interaction (Liddicoat 2011), la construction de l'ethos en conversation en ligne (Constantin de Chanay 2011), l'accentuation des mimiques faciales et des comportements gestuels par rapport à une situation de face à face présentiel (Cosnier, Develotte 2011). On notera que la conclusion de Décrire la conversation en ligne ${ }^{3}$ considérait que le champ scientifique était prêt à prendre à bras-le-corps ces nouveaux types de données en développant des méthodologies fondées sur des complémentarités interdisciplinaires (par exemple, conversation, environnement, espaces) et sur des renouvellements formels incluant l'intégration de vidéos pour établir des standards de recherche plus rigoureux, et mieux rendre compte des éléments tels que les gestes, les mimiques, les regards, les postures (2011, p. 194-195).

\section{Les apports des sciences de l'information et de la communication}

Dès la fin des années 1990 en France, les SIC examinent les nouvelles formes de l'écrit issues de l'usage d'appareils informatiques et d'internet et proposent des dispositifs théoriques et une terminologie qui restent porteurs dans les travaux actuels. On mentionnera ici les notions d'écrit d'écran, d'énonciation éditoriale, d'hypertextualité et d'identité numérique.

\subsection{Les écrits d'écran}

En 1996, dans l'article devenu classique «L'écrit d'écran, pratiques d'écriture \& informatique », Emmanuël Souchier souligne les modifications apportées par l'informatique : "Nous assistons à différents types de modifications portant essentiellement sur la matérialité et les supports, l'acte et les pratiques d'écriture, sur les partenaires de l'écrit, sur la division du travail, sur le temps, l'espace et la diffusion de l'écrit » (1996:106). Pour lui, le passage du papier à l'écran et du stylo au clavier implique trois changements majeurs : une dématérialisation du texte puisque la trace des lettres inscrites sur un support devient sur

\footnotetext{
${ }^{3}$ On précisera que cet ouvrage part des outils méthodologiques « classiques » pour en tester la validité, et surtout les limites, sur la conversation en ligne, dans une perspective similaire à celle du sociolinguiste Androutsopulos qui revisite la notion d'hétéroglossie (Bakhtine) pour en montrer la complexité appliquée aux médias sociaux. Le propos de cet article est de montrer qu'un pas en avant a été fait depuis ces travaux et que nouveaux concepts et notions spécifiquement adaptés aux réalités étudiées ont émergé et sont en train de nourrir les réflexions circulant dans le domaine des discours numériques.
} 
l'écran une trace électronique immatérielle et fugitive ; une modification dans la dimension corporelle de l'écriture, la main n'ayant plus de contact direct avec l'écrit mais devenant un outil de frappe sur clavier ; une double abstraction, celle du texte se trouvant augmentée de celle de l'écrit qui n'existe plus que virtuellement sous la forme de lignes de code. L'auteur considère que l'écran est le nouvel espace où s'élabore toute pratique d'écriture informatique, espace qui s'inscrit dans l'histoire continue des outils d'écriture et qui présente des traits spécifiques. Cette notion d'écrit d'écran a été largement reprise, notamment par Yves Jeanneret, qui en montre la capacité plastique; l'écran permet en effet les "métamorphoses » de l'écrit, devenu manipulable et transformable grâce aux outils technologiques (Jeanneret 2000).

\subsection{L'énonciation éditoriale}

Emmanuël Souchier propose également la notion d'énonciation éditoriale, décrivant un mode d'élaboration pluriel du texte, marqué par une forme de polyphonie énonciative (intervention de plusieurs instances humaines et non humaines), une hybridation multimédiatique (texte, image fixe ou animée, son), le caractère labile et transformable du texte et ses possibilités de circulation inédites (Souchier 1998). L'énonciation éditoriale " désigne l'ensemble de ce qui contribue à la production matérielle des formes qui donnent au texte sa consistance, son "image de texte". [...] Plus fondamentalement, l'énonciation éditoriale est ce par quoi le texte peut exister matériellement, socialement, culturellement... aux yeux du lecteur » (Jeanneret, Souchier 2005: 6). Cette notion n'a pas été intégrée par les premières approches linguistiques des productions électroniques/numériques, restant concentrées sur les données langagières au détriment peut-être de leurs mises en forme matérielles.

\subsection{L'hypertextualité}

L'hypertextualité, phénomène central affectant tout discours connecté, est prise en compte depuis les années 1990 en SIC (par exemple Clément 1995) mais également en littérature (voir l'importante synthèse de Saemmer 2015), les SDL ne s'étant que très récemment saisies de ce phénomène considéré comme ne relevant pas vraiment de son périmètre (Simon 2016 dir.). L'hypertextualité est le mécanisme qui consiste à permettre, à partir d'un texte ou d'un énoncé source, de se diriger vers un texte ou un énoncé cible, via ce que Serge Bouchardon appelle un " énoncé de geste », c'est-à-dire le cliquage sur un hyperlien (Bouchardon 2011). Les travaux sur l'hyperlien sont encore trop rares, comme le signale Alexandra Saemmer : "Alors que les approches rhétoriques du texte papier remplissent des bibliothèques entières, l'étude sémiotique et rhétorique du texte numérique et de ses spécificités sur la page-écran reste encore un champ de recherche peu défriché » (2015: 36). On peut en dire autant de son étude linguistique, alors même que les travaux des littéraires ou des spécialistes des SIC ont toujours posé des questions aux implicites linguistiques forts. Qu'il s'agisse des écrits sur la littérature informatique, des travaux sur le texte numérique (Bouchardon, Clément), sur l'hypertexte lui-même (Saemmer) ou du paradigme des écrits d'écran (Souchier, Jeanneret), tous les chercheurs ont posé des questions interrogeant fondamentalement la langue, l'élaboration du texte et du discours, et la construction du sens par les agents.

\subsection{L'identité numérique}

Les travaux développés autour de l'identité numérique par Fanny Georges notamment $(2009,2011)$ viennent informer les recherches effectuées en analyse du discours sur les dimensions énonciatives des discours ou, dans le domaine des interactions, sur les comportements des interactants par écran. Les trois dimensions constitutives de l'identité numérique qui sont selon sa terminologie, l'identité déclarée (la façon de se présenter), l'identité agissante (les actes que nous faisons) et l'identité calculée (présence au niveau informatique), possèdent en effet leurs déclinaisons spécifiques dans tous les lieux 
d'énonciation numérique, qu'il s'agisse des différentes plateformes du web 2.0, des plateformes synchrones de visioconférence poste à poste ou encore des forums asynchrones. L'identité déclarée est très liée à l'imaginaire et rejoint les travaux de Ghislaine Chabert qui a travaillé sur l'espace de l'écran. Elle distingue « trois environnements associés à l'écran: l'espace physique (bureau et environnement de chaque personne), l'espace imaginé (hors champ de l'autre à distance) [...] et l'espace de l'écran (espace surface, espace touché) (2012 : 210). Ces trois environnements font de l'écran un «lieu frontière, d'entre deux mondes, [il] articule des espaces hétérogènes, des espaces dans l'écran et aussi des espaces hors écrans " (Chabert 2012 : 210). Pour le philosophe Stéphane Vial (2013) également chercheur en SIC, qui étudie «comment le numérique change la perception " (sous-titre de son ouvrage), la révolution numérique est un événement phénoménologique qui affecte notre expérience du monde et qui relève de l'ontologie, ou plutôt de l'ontophanie, c'est-à-dire de la manière dont les êtres (ontos) apparaissent (phaïno) (2013 : 98). Pour lui, « la technique est une structure de la perception » (2013 : 99). "Être natif du numérique, explique-t-il, c'est avoir acquis la faculté de voir apparaître le monde en étant numériquement appareillé » (2013 : 146). La présence à distance par écran est donc configurée par l'apparition et la posture à l'écran, et d'une nature spécifique par rapport à celles que nous connaissons dans les relations présentielles.

On pourrait s'interroger sur l'absence de la sociologie dans le tableau que nous venons de brosser. Mais cette discipline, qui a pourtant très largement parcouru les cultures et les sociabilités numériques, ne semble pas prendre en compte les aspects langagiers, sociolangagiers ou discursifs, se concentrant sur les productions en ligne sous l'angle des cultures numériques (par exemple Casilli 2011) ou des pratiques politiques (par exemple Cardon 2010). Quand les sociologues envisagent les traces ou les données numériques notamment, ils privilégient les approches communicationnelles et quantitatives et écartent les aspects langagiers; il est notable que les travaux soient alors publiés dans des revues de SIC (voir par exemple Venturini et al. 2015).

\section{Questions posées à la linguistique par les productions numériques}

À partir de la situation décrite dans les deux parties précédentes, nous nous proposons de formuler quelques questions linguistiques posées par le recours aux dispositifs numériques dans les communications et relations humaines, et d'en montrer les conséquences sur les configurations théoriques et méthodologiques en sociolinguistique et dans les disciplines TDI.

\subsection{La nature des productions langagières}

Quelle est la nature des mots, des textes et des discours qui s'élaborent en ligne? Autrement dit, la linguistique peut-elle conserver, pour décrire les discours natifs de l'internet, la description du langage héritée du $X X^{e}$ siècle ? Nous pensons que, pour s'aventurer sur les terrains du numérique, les linguistes doivent modifier leurs équipements théoriques et méthodologiques.

En ligne, les données langagières ne sont pas constituées de matières purement langagières, mais sont composites, métissées de non-langagier de nature technique. Cette nature hybride est manifeste dans certaines formes comme les hashtags marqués du croisillon, les pseudos du réseau Twitter marqués par l'arobase, ou encore tous les segments rendus cliquables par des hyperliens, qui marquent d'ailleurs leur hybridité par la couleur et/ou le soulignement, phénomène inédit hors ligne (Paveau 2013). D'une manière plus générale, l'ensemble des éléments langagiers et discursifs qui sont produits en ligne sont dotés de ce caractère composite du fait même de la nature de leur écosystème de production : la réticularité d'internet construite par les algorithmes implique en effet que tous les énoncés en ligne constituent des liens; la relationalité, on le verra plus bas, est en effet un trait structurant des discours numériques natifs. 
L'idée d'une matière langagière composite suppose une approche non dualiste des productions verbales qui remet en cause la distinction binaire entre linguistique et extralinguistique (Brassac 2004). Cette approche suppose des liens entre l'humain et le nonhumain qui dépassent la simple utilisation des objets pour envisager des réalités véritablement hybrides. Dans les univers discursifs numériques, l'hybridité est principalement celle qui assemble du discursif et du technique: les discours numériques natifs sont des technodiscours (Paveau 2013). Ce type de description est également à l'œuvre dans le cadre des interactions médiées par écrans : la notion d'espace d'exposition discursive des médias sociaux proposée par Christine Develotte (2009) renvoie à la matérialité technosémio-linguistique de leur support en ligne, aussi bien à leur design sur l'écran, qu'aux fonctionnalités qu'ils proposent. II s'agit d'espaces multimodaux ${ }^{4}$ faisant co-exister des éléments graphiques, iconiques et picturaux, statiques ou mobiles, et des fonctions de communication synchrones et asynchrones. Ces espaces discursifs conditionnent d'une part ce qu'il pourra être possible d'énoncer et de produire dans l'interaction. Par exemple, sur Facebook 2017, la modalisation associée à la fonction " commentaire » des interactions par les émoticônes, s'est récemment enrichie mais reste limitée à six possibilités, et la netiquette qui s'applique sur la plateforme évacue les propos jugés trop violents ou déplacés. D'autre part, c'est l'utilisateur qui choisit, en fonction de ses souhaits et de ses interlocuteurs, sa façon d'utiliser ou non, de combiner ou non toutes les possibilités que le système offre, tout ce qui le caractérise en tant qu'individu : remplir ou non son profil sur Facebook dans la partie proposée, poster une vidéo avec ou sans commentaire, commenter par un texte langagier, par une émoticône, une photo, une vidéo ou une combinaison de différents moyens, écrire ou parler par "message privé ", etc. Les médias sociaux sont donc un espace d'exposition discursive qui configure les productions discursives qui pourront avoir lieu en fonction de leurs affordances (Gibson 1979).

Si l'on veut rendre compte des discours natifs d'internet, il faut donc adopter une perspective qui dépasse l'idée d'une séparation entre le langage et ses extérieurs, en particulier techniques, et modifier la conception de la langue pour la penser comme coconstituée d'une altérité technique.

\subsection{La dimension relationnelle des productions numériques}

Les productions numériques, du fait de la structure hypertextuelle d'internet, et en particulier du web, sont des énoncés délinéarisables, augmentables et innombrables. Ces traits constituent des défis pour l'analyse linguistique.

Délinéarisables. À l'écrit, les hyperliens délinéarisent le discours, tant en production qu'en réception, en écriture qu'en lecture, les deux processus étant d'ailleurs étroitement imbriqués (sur un blog ou un journal en ligne par exemple, le lecteur écrit le texte à mesure qu'il le lit en cliquant sur les liens proposés, c'est le phénomène d'écrilecture ${ }^{5}$ ). Dans la communication par écrans, la simultanéité de l'affichage de plusieurs écrans sur une même page délinéarise une conversation que l'analyse conversationnelle classique a l'habitude de transcrire linéairement (Develotte et al. 2011 dir). La délinéarisation se produit également dans les jeux vidéo : reprenant l'idée d'« espaces hybrides », Marc Relieu (2005) ainsi qu'Heike Baldauf et Isabelle Colon de Carvajal (2015) ont étudié les interactions entre joueurs lors de parties de jeux vidéo. Ils montrent que la coprésence des avatars et des joueurs amènent à des " hybrides » et à des indéterminations dans l'identification du destinataire des énoncés, à ce que nous pourrions appeler des "énoncés dédoublés », qui s'adressent aussi bien à l'avatar de l'adversaire qu'à l'adversaire lui-même.

Augmentables. Le web 2.0, dit « conversationnel », permet le commentaire sur les espaces des blogs et des RSN. Toute intervention est alors augmentée de remarques ou de conversations, ce qui pose des problèmes de circonscription discursive et textuelle:

\footnotetext{
${ }^{4}$ Nous regroupons ici la multimodalité au sens propre (utilisation de différents modes d'expression) et la multimédialité (utilisation de différents médias) sous un même terme. La différenciation ne nous semble pas appropriée pour nos interrogations ici.

${ }^{5}$ Sur les phénomènes de délinéarisation et d'écrilecture dans le cadre de l'hypertextualité, voir Paveau 2016.
} 
comment définir un billet de blog ? Avec ou sans les commentaires ? Comment définir un post ou un tweet ? Avec ou sans les réponses et les partages ? De la réponse apportée à cette question découlera une autre question centrale: qui est l'énonciateur? Comment identifier un auteur? Le problème s'intensifie dans certains espaces d'écriture comme les pads, permettant à plusieurs scripteurs de collaborer au même document, simultanément, chacun marqué par une couleur qui marque une polyphonie qui n'en est pas une : c'est bien sur le même fil de discours que les scripteurs écrivent, produisant au bout du compte un texte unique, tournant parfois à l'énigme énonciative : comment traiter la question de l'ethos dans ce cas?

Innombrables. Les énoncés numériques natifs sont doublement innombrables: d'une part, contrairement aux énoncés hors ligne (ouvrages, journaux, échanges oraux), les énoncés numériques natifs ne connaissent pas de clôture, toujours transformables, par délinéarisation ou augmentation notamment ; d'autre part, internet étant un univers relationnel, et le web social permettant la fonction de partage, tout énoncé est susceptible d'être remis en circulation dans d'autres contextes que son contexte d'origine, et d'y être de nouveau délinéarisé ou augmenté. Prenons l'exemple d'une vidéo d'un vlogueur sur Youtube : il publie une vidéo sur sa chaîne Youtube, mais simultanément sur son site, son compte Facebook, son compte Twitter et son compte Instagram. Cette publication primaire entraîne une quantité innombrable d'énoncés secondaires : les modes de présentation sur chacun de ces écosystèmes ne sont pas forcément les mêmes (formats, textes de présentation, images affichées) ; chaque écosystème reçoit des commentaires, et pour des vlogueurs comme Norman ou Cyprien par exemple ${ }^{6}$, il s'agit de plusieurs milliers ; sur chaque plateforme, des partages s'effectuent, vers les comptes personnels ou les pages des internautes sur les RSN, à partir desquels des partages s'effectuent de nouveau, déclenchant d'autres commentaires, etc.

L'ensemble de ces traits constitue un véritable questionnement posé à la linguistique tant sur le plan théorique que méthodologique.

\subsection{La construction des corpus}

Des caractéristiques précédentes découlent des questionnements concernant le recueil des données, l'élaboration des observables et la construction des corpus. Des données langagières marquées par l'innombrabilité, l'augmentabilité, la variabilité et l'hypertextualité constituent un défi pour le linguiste : comment constituer un corpus de technodiscours 2.0, sur les RSN notamment? Faut-il ne sélectionner que les énoncés primaires ou intégrer les énoncés secondaires ? Que faire des milliers de commentaires qui augmentent parfois les énoncés primaires? Doit-on tous les traiter? Ou les sélectionner? Si oui, selon quels critères ? Comment, de même, constituer des corpus d'interactions médiées par écrans multiples ?

La difficulté de répondre à ces questions est redoublée par une autre caractéristique encore insuffisamment intégrée: la subjectivité intrinsèque des données du web 2.0 et des conversations par écrans. En effet, ce que l'internaute voit, entend et lit sur son écran, tout particulièrement sur les RSN, est contraint par la manière dont il configure son navigateur, par les accès à ses comptes et les autorisations qu'il programme, et par les prescriptions des algorithmes produisant des informations à partir de ses traces. Cela veut dire que ces corpus numériques natifs ne peuvent être traités comme l'étaient le plus souvent les corpus prénumériques, par exemple les discours de presse, les discours littéraires ou même les conversations. Ceux-ci possèdent des formes stabilisées, closes et objectives (un article de presse est le même pour tout le monde), contrairement aux données numériques qui sont, comme le précise Laetitia Émerit, instables, mixtes et incomplètes. "Renoncer à cette instabilité, explique-t-elle, c'est perdre la nature technolangagière des données pour ne conserver qu'une image de celles-ci. C'est aussi renoncer à l'interactivité que permettent ces

6 Norman fait des vidéos: https://www.youtube.com/user/NormanFaitDesVideos ; Cyprien : https://www.youtube.com/user/MonsieurDream 
objets de recherche en les isolant des locuteurs-scripteurs-utilisateurs et de leur écosystème numérique d'apparition (Émerit 2016: § 22). Elle propose donc, à partir de la notion de "corpus idionumérique " (c'est-à-dire lié à la subjectivité du seul internaute), une méthodologie permettant de rendre compte de ces traits spécifiques. La notion de "lieu de corpus ", définie comme un "espace numérique délimité dont les données possèdent trois caractéristiques incompatibles avec la notion de corpus : l'instabilité, la mixité et l'incomplétude ", lui permet d'élaborer une représentation arborescente des corpus du web 2.0 de manière à intégrer leurs six caractéristiques: multimodalité, ouverture, évolutivité, interactivité, interconnexion et idionuméricité.

\section{Analyser les discours numériques natifs}

Nous considérons, dans une perspective macro, que les dispositifs techniques, loin de ne constituer que des supports à la production des discours, sont de véritables milieux pour les énoncés natifs en ligne, écrits et oraux: ceux-ci constituent en effet des composites assemblant technique et langagier, susceptibles par exemple d'une analyse en termes d'affordances (Develotte 2012). Dans les perspectives meso et micro, cela veut dire que les dispositifs numériques de communication écrite et orale et les formes sociolangagières et sociorelationnelles spécifiques de l'internet (spécifiques n'impliquant pas forcément inédites) se coproduisent, configurant des pratiques sociodiscursives numériques qui signent des modes langagiers, communicationnels et relationnels ayant intégré les paramètres technologiques (Paveau 2015).

\subsection{Interactions multimodales par écrans. L’ajustement dans la visioconférence}

Les travaux menés dans le champ des interactions multimodales par écrans (IMPEC ${ }^{7}$ ) ont montré que les notions couramment utilisées par les interactionnistes devaient être revisitées pour devenir opératoires sur les discours numériques natifs.

Si l'on prend l'exemple de la conversation par visioconférence poste à poste (de type Skype), le seul fait que l'on ait, à l'écran, sa propre image, en plus de celle de l'interlocuteur, implique différents ajustements (Develotte et al. 2011 dirs). Samira Ibnelkaïd, montrant comment fonctionne la rencontre en ligne dans un contexte situé, c'est-à-dire l'événement de la mise en relation de deux personnes par le biais de la visioconférence poste à poste, revisite certaines notions proposées par Goffman, comme le décor ou la façade (Ibnelkaïd 2016). Elle montre que dans un tel dispositif il y a dédoublement du décor et de la façade, l'un étant primaire (le décor dans lequel a lieu l'interaction) et l'autre secondaire (celui qui est renvoyé par l'image sur l'écran). II s'agit donc d'une médiation technique complexe qui construit un dispositif de façades et décors secondaires (Ibnelkaïd 2015) et induit une énonciation spécifique qui peut être explicitée ainsi : c'est mon image (ma «façade secondaire » pour reprendre les termes de Samira lbnelkaïd) qui parle à l'image, à la "façade secondaire " de mon interlocuteur. Suis-je d'accord avec ce que cette façade renvoie de moi et de ce que je veux dire?

Pour répondre à cette question, différents ajustements s'avèrent nécessaires, en amont et pendant le déroulement de la conversation par écran :

- En amont de l'interaction : les interactants se préparent à l'avance, par exemple en vérifiant l'image de soi qu'ils veulent ou non transmettre (différentes stratégies sont possibles pour éviter de se montrer: je n'ai pas de webcam, ma vidéo ne marche pas, etc.). Ils accomplissent également des ajustements aux contraintes posturales (nécessité d'être centré par rapport à l'écran, fixité, regard dirigé vers l'interlocuteur) qui influencent leurs comportements respectifs.

\footnotetext{
${ }^{7}$ Le groupe de travail IMPEC rattaché au laboratoire ICAR mène depuis plusieurs années des recherches sur les interactions par écrans en partant d'ancrages disciplinaires différents (principalement SDL, sciences cognitives et $\mathrm{SIC)}$ : https://impec.sciencesconf.org/resource/page/id/23.
} 
- Pendant l'interaction: les interactants s'adaptent ou non à l'image de soi par des régulations, des améliorations de leur comportement verbo-posturo-mimo-gestuel, une gestion des tours de regard à soi-même. Ils modifient également leurs comportements " normaux " pour ne pas " gêner » la conversation en ligne, ne pas introduire de doute dans l'esprit de l'interlocuteur en cas de changement de direction de regard par exemple: ils peuvent par exemple chercher à donner à l'interlocuteur l'illusion qu'on le regarde dans les yeux (Develotte 2012). Ils peuvent aussi, lors de multi-tasking, fumer une cigarette " en cachette " ou écrire " à l'aveugle », toujours afin de ne pas troubler l'échange. De la même manière, ils peuvent se détacher de l'écran (pour vivre normalement, manger ensemble, etc.) ou se détacher de la fonction visuelle en ôtant la fonction visio (Kozar 2015). Tous ces comportements varient selon les interlocuteurs avec lesquels on échange, familiers ou inconnus (Jauréguiberry 2000), la situation professionnelle, commerciale, familiale, amoureuse.

- En aval de l'interaction : selon la tension et la durée de la communication, on peut repérer une certaine fatigue après l'échange par écran pouvant aller jusqu'à un choc dépressif quand l'échange visuel en ligne a par exemple ravivé la douleur de l'éloignement.

\subsection{Analyse du discours numérique. La délimitation des unités}

Une des questions cruciales des SDL est la délimitation et l'identification des unités de travail de la discipline. On a vu plus haut que, dans le cas des énoncés numériques natifs, qu'il s'agisse des énoncés écrits ou des interactions orales, la question de l'identification se reposait, et qu'il fallait envisager des unités langagières hybrides, autrement dit technolangagières, la matière langagière numérique étant composite. Les technodiscours posent en outre de redoutables problèmes de délimitation, qu'un applicationnisme prénumérique trop rapide laisse souvent de côté. Les énoncés numériques natifs écrits dans les contextes du web 2.0 sont largement dépendants des CMS (Content manager systems, ou systèmes de gestion de contenus) et des templates (modèles de pages) qu'ils proposent. Les formats éditoriaux impliqués par ces contraintes (qui ressortissent typiquement à l'énonciation éditoriale) sont souvent confondus avec leur forme énonciative : sur le réseau Twitter par exemple, le tweet est souvent défini comme une suite de 140 signes, alors qu'il s'agit d'un format ; sur Facebook, le post est défini pareillement comme une publication dans le cadre de la fenêtre "Créer une publication "; sur Youtube, la vidéo sera réduite à sa matérialité filmique.

Dans ces trois exemples, on doit pourtant faire une distinction entre format et forme, et prendre en compte la production technodiscursive dans son environnement natif, qui, comme on l'a vu plus haut, la rend délinéarisable, augmentable, innombrable et investigable, essentiellement du fait de la structure hypertextuelle du web. Identifier un tweet comme la seule suite de 140 signes et en faire la base d'une recherche pose d'importants problèmes de validation scientifique : comme la phrase entre la majuscule et le point, le tweet ne se laisse pas enfermer dans un format quantitatif, mais se développe hors des 140 signes via différents processus produsés ${ }^{8}$ par les internautes: le franchissement pur et simple par l'énoncé de la limite quantitative, la suite de tweets numérotés, le thread (autoréponse filée, permise par la plateforme depuis 2014). Les réponses au tweet simple ou filé, sa situation dans le fil d'abonné (le tweet figure par définition dans le fil de tous les abonnés d'un compte, ses contextes sont donc multiples), ses circulations par retweet ou partages privés, ses intégrations sur d'autres plateformes (des blogs ou des articles de presse par exemple) sont par ailleurs autant d'éléments qui en font un énoncé structurellement relationnel, non isolable pour une analyse hors contexte. Enfin, un tweet est doté de plusieurs métadonnées qui participent pleinement de son fonctionnement sémantique et discursif : statut de tweet, retweet ou réponse, date et heure, nombre de favoris, nombre de retweets. Comme les mots isolés, les tweets isolés sont des fictions linguistiques, et numériques de surcroît. Le même

\footnotetext{
${ }^{8}$ Le produsage (mot-valise constitué à partir de production + usage) désigne selon Axel Bruns le processus conjoint d'utilisation et de modification des affordances numériques du web (Bruns 2008).
} 
type de description peut être fait du post sur Facebook ou un autre réseau social comme Linkedln ou Pinterest, de la vidéo sur les plateformes visuelles: la relationalité de l'écosystème du web implique en effet que les énoncés y soient des liens. C'est une dimension nouvelle qui doit être intégrée dans les équipements théoriques et méthodologiques des linguistes en terrain numérique.

\section{Conclusion}

Nous avons voulu montrer dans cet article que les discours numériques natifs posaient d'importants et passionnants problèmes aux linguistes et à la linguistique elle-même dans ses modalités d'identification et de délimitation des unités, ses méthodes de recueil de données et d'élaboration de corpus, ainsi que ses dispositifs d'analyse des productions langagières. La relationalité des énoncés en ligne, leur innombrabilité, leur multimodalité, leur évolutivité et ce qu'on peut appeler leur ubiquité constituent des défis que les sciences du langage doivent selon nous relever en adoptant des perspectives non dualistes, intégrant l'humain et le non-humain, le linguistique et le technologique. Nous pensons qu'il y a place actuellement pour d'autres épistémologies, d'autres méthodes d'analyse à créer en prenant appui sur les disciplines en SHS de façon à mieux prendre en compte la complexité technosémio-discursive des discours numériques.

\section{Références bibliographiques}

N.B. Tous les liens ont été vérifiés le 21 décembre 2016

Amossy, R. (2011), "La coexistence dans le dissensus", Semen, 31, http://semen.revues.org/9051

Anis J. (1998), Texte et ordinateur. L'écriture réinventée? Paris-Bruxelles, De Boeck Université.

Baldauf-Quilliatre H. \& Colón de Carvajal I. (2015), "Is the avatar considered as a participant by the players? A conversational analysis of multi-player videogames interactions ", PsychNology Journal, 13- 2-3, p. 127-147.

Baron N., (2000), Alphabet to E-mail: How Written English Evolved and Where It's Heading, London, New York, Routledge.

Bouchardon S. (2011), « Des figures de manipulation dans la création numérique », Protée 39, p. 37-46.

Brassac C. (2004), «Action située et distribuée et analyse du discours: quelques interrogations » Cahiers de linguistique française 26, p. 251-268.

Bruns A. (2008), Blogs, Wikipedia, Second Life, and Beyond. From Production to Produsage, Bern, Peter Lang.

Calabrese L. (dir) (2011), "L'internet, corpus sauvage. Nouvelles ressources, nouveaux problèmes ? ", Le discours et la langue 2.1.

Cardon D. (2010), La démocratie Internet. Promesses et limites, Paris, Éditions du Seuil.

Casilli A. (dir) (2011), Communications 88, dossier « Cultures du numérique ».

Chabert G. (2012), « Les espaces de l'écran », Écrans et Médias 34, p. 203-215.

Constantin de Chanay H. (2011), "La construction de l'éthos dans les conversations en ligne », dans Develotte C., Kern R. \& Lamy M.-N. (dirs) (2011), Décrire la conversation en ligne. Le face à face distanciel, Lyon, ENS Éditions, p. 145-172.

Cosnier J. \& Develotte C., "Le face à face en ligne : approche éthologique », dans Develotte C., Kern R. \& Lamy M.-N. (dirs) (2011), Décrire la conversation en ligne. Le face à face distanciel, Lyon, ENS Éditions, p. 27-50.

Crystal David, 2001, Language and the Internet, Cambridge, CUP.

Develotte C. (2009), " From face to face to distance learning: the online learner's emerging identity ", in Goodfellow, R. \& Lamy, M.N., (eds), Learning Cultures in Online Education, London, Continuum Books, p. 71-92. 
- (2012), "L'analyse des corpus multimodaux en ligne : état des lieux et perspectives », Congrès Mondial de Linguistique Française - CMLF 2012 http://dx.doi.org/10.1051/shsconf/20120100213

Develotte C. \& Lancien T. (2000) «Propositions pour l'analyse des discours multimédia : l'exemple de deux articles encyclopédiques », dans Lancien, T. (dir.), Multimédia: les mutations du texte, Fontenay-aux-Roses, ENS Editions, p. 119-139

Develotte C., Kern R. \& Lamy M.-N. (dirs) (2011), Décrire la conversation en ligne. Le face à face distanciel, Lyon, ENS Éditions.

Émerit L. (2016), « La notion de lieu de corpus: un nouvel outil pour l'étude des terrains numériques en linguistique », Corela 14-1, http://corela.revues.org/4594

Fraenkel B. \& Marcoccia M. (dirs), 2003, «Écrits électroniques : échanges, usages et valeurs », Langage et société 104.

Georges F. (2009), « Représentation de soi et identité numérique. Une approche sémiotique et quantitative de l'emprise culturelle du web 2.0 », Réseaux 154, p. 165-193.

- (2011), "L'identité numérique sous emprise culturelle. De l'expression de soi à sa standardisation ", Les cahiers du numérique 7-1, p. 31-48.

Gibson J.J. (1979), The Ecological Approach to Visual Perception, Hillsdale, Lawrence Erlbaum.

Herring S. (ed.), 1996, ComputerMediated Communication : Linguistic, Social and CrossCultural Perspectives. Pragmatics and Beyond series. Amsterdam, John Benjamins.

- (2004), "Online communication: Through the lens of discourse », in M. Consalvo, N. Baym, J. Hunsinger, K. B. J'enceins, J. Logie, M. Murero, \& L. R. Shade (eds), Internet Research Annual, volume I, New York, Peter Lang, p. 65-76.

lbnelkaïd S. (2015), "Scénographie d'une ouverture d'interaction vidéo », Réseaux 194, p. 125-168.

- (2016), Identité et altérité par écran: modalités de l'intersubjectivité en interaction numérique, thèse de doctorat, Université Lumière Lyon 2.

Jauréguiberry F. (2000), "Le moi, le soi et Internet », Sociologie et sociétés 32-2, p. 136152.

Jeanneret Y. (2000), Y a-t-il (vraiment) des technologies de l'information? Lille, Presses Universitaires du Septentrion.

Jeanneret Y. \& Souchier E. (2005), "L'énonciation éditoriale dans les écrits d'écran », Communication et langages 145, p. 3-15.

Kozar O. (2015), «Language Education via Video/Audio Conferencing : a Discursive Investigation », Linguistics and Education 31, p. 86-100

Liddicoat A.J. (2011), «Enacting participation: hybrid modalities in online video conversation ", dans Develotte C., Kern R. \& Lamy M.-N. (dirs) (2011), Décrire la conversation en ligne. Le face à face distanciel, Lyon, ENS Éditions, p. 51-70.

Liénard F. \& Zlitni S. (dirs) (2015), La communication électronique: enjeux, stratégies, opportunités, Limoges, Lambert-Lucas.

- 2015, "Communication numérique ou communication électronique ? ", dans Liénard F. \& Zlitni S. (dirs), La communication électronique: enjeux, stratégies, opportunités, Limoges, Lambert-Lucas, p. 8-19.

Maingueneau D., 2013, "Genres de discours et web : existe-t-il des genres web ? », dans Barats C. (dir.), 2013, Manuel d'analyse du web en sciences humaines et sociales, Paris, Armand Colin, p. 74-91.

Marcoccia M. (2011), «'T'es où maintenant ?' : les espaces de la conversation visiophonique en ligne », dans Develotte C., Kern R. \& Lamy M.-N. (dirs) (2011), Décrire la conversation en ligne. Le face à face distanciel, Lyon, ENS Éditions, p. 95-115.

Mondada L., (1999), "Formes de séquentialité dans les courriels et les forums de discussion », ALSIC 2-1, p. 3-25.

Mourlhon-Dallies F., Rakotonoelina F., Reboul-Toure S. (dirs), 2004, « Les discours de l'internet : quels enjeux pour la recherche? ", Les Carnets du Cediscor 8. 
Panckhurst R. (1998), "Marques typiques et ratages en communication médiée par ordinateur ", Actes du colloque CIDE 98, INPT, Rabat, 15-17/04/98, Paris, Europia Productions, p. 31-43.

Paveau M.-A. (2013), « Technodiscursivités natives sur Twitter. Une écologie du discours numérique », Epistémè 9, p. 139-176.

- (dir.) (2015), Itinéraires Itc, dossier «Textualités numériques », https://itineraires.revues.org/2258

- (2015), «Ce qui s'écrit dans les univers numériques », Itinéraires Itc., http://itineraires.revues.org/2313

- (2016), "Des discours et des liens. Les parcours technodiscursifs de l'écrilecture », Semen 42, p. 23-48.

Pierozak I. (2003), Le français tchaté. Une étude en trois dimensions - sociolinguistique, syntaxique et graphique - d'usages IRC, thèse de doctorat, Université d'Aix-Marseille I.

- (2010), "Les corpus électroniques en sciences du langage : un eldorado ? ", Le discours et la langue 2.1, p. 15-32.

Relieu M. (2005), "Les usages des TIC en situation naturelle: une approche ethnométhodologique de l'hybridation des espaces d'activité », Intellectica 41-42, p. 139-162.

Saemmer A. (2015), Rhétorique du texte numérique. Figures de la lecture, anticipations de pratiques, Villeurbanne, Presses de l'Enssib.

Souchier E. (1996), « L'écrit d'écran, pratiques d'écriture \& informatique », Communication et langages 107, p. 105-119.

- (1998), "L'image du texte. Pour une théorie de l'énonciation éditoriale ", Cahiers de médiologie 6, p. 137-145.

Traverso V. (2011), "Chevauchements de parole, résolution et réparation dans la conversation en ligne ", dans Develotte C., Kern R. \&Lamy M.-N. (dirs), Décrire la conversation en ligne. Le face à face distanciel, Lyon, ENS Éditions, p. 117-143.

Vial S. (2013), L'être et l'écran. Comment le numérique change la perception, Paris, Puf. Venturini T., Cardon D. \& Cointet J.-P. (dirs) (2015), " Méthodes digitales. Approches quali/quanti des données numériques », Réseaux 188. 